\title{
De los riesgos en la socialización global: adolescentes en conflicto con la ley con perfil de ajuste social
}

\author{
On the risks of global socialization: adolescents in conflict with the law \\ with social adjustment profile
}

\author{
José Javier Navarro-Pérez / J.Javier.Navarro@uv.es \\ http://orcid.org/0000-0001-6363-7154 \\ Universidad de Valencia, España \\ Enrique Pastor-Seller / epastor@um.es \\ http://orcid.org/0000-0001-8693-5138 \\ Universidad de Murcia, España
}

\begin{abstract}
This article explains the consequences of social change for the adolescents of the new era. This research has different methods and techniques to triangulate the evidence. The study involved 183 adolescent offenders legally incarcerated for a period of five years (as of 2011). The main results report that these adolescents live in protected contexts. Parents are responsible for an education poor in values. The depersonalization of human beings is reported. There is little critical and creative and virtual leisure aimed at adolescents. The findings report a society that has lost the essence of community. The risk is daily and visible in all social classes.
\end{abstract}

Key words: socialization styles, adolescents in conflict with the law, risks, developed societies, social adjustment, delinquency.

Resumen: El presente artículo analiza los efectos del cambio social en los adolescentes de la era del riesgo. Se trata de una investigación que integra diferentes métodos y técnicas en aras de triangular las evidencias. Se estudiaron 183 adolescentes condenados a medidas de internamiento judicial durante un periodo de cinco años (desde 2011). Como principales hallazgos destacan la presencia de riesgo en contextos protegidos, donde los padres han disminuido el compromiso instructivo sobre sus hijos, delegando su responsabilidad parental, reduciéndose así la educación familiar en valores prosociales. Por otro lado, se alerta en torno a la cosificación de las relaciones entre las personas y la existencia de un ocio despersonalizado y virtual que paraliza la actitud crítica y creativa de los adolescentes. Las conclusiones muestran una sociedad altamente individualizada, donde el riesgo emerge como un aspecto en la vida cotidiana y presente en todos los círculos y estratos sociales.

Palabras clave: estilos de socialización, adolescentes en conflicto con la ley, riesgos, sociedades desarrolladas, ajuste social, delincuencia. 


\section{Introducción}

El desarrollo humano se construye sobre una base de inseguridad que nunca antes se había producido. Respecto a los desastres sobrevenidos en las nuevas sociedades contemporáneas, Benavides (2013) considera que las actuales formas de vida están teledirigidas. Se produce un desgaste de ideales, el triunfo de la individualización y la ruptura con la tradición; hechos que influyen en la vida colectiva.

El presente artículo analiza los efectos del cambio social en adolescentes a priori ajustados a su contexto; nos aproximaremos a su cotidianidad, a sus sistemas de relación periférica y en cómo los tentáculos de la llamada sociedad del riesgo (Beck, 1992) influyen en sus rutinas y les empujan a comportamientos penalmente sancionables.

\section{Prosperidad incierta: bienestar y socialización de los adolescentes}

La sociedad tecnológica redunda en un escenario donde las posibilidades de crecimiento permiten construir el imaginario colectivo con muchas esperanzas. Los adolescentes han nacido en el contexto de compra-venta. En esta opulencia a la que Galbraith (1997) hacía referencia, han nacido y crecido las nuevas generaciones de adolescentes. El consumo se convierte en argumento, y la cultura del placer en principal atributo (Berger y Huntington, 2002). Una sociedad insaciable que devora las novedades.

Lo efímero pasa a ser un valor de primera necesidad. Desde esta perspectiva, Lipovetsky (2007) cimienta su teoría sobre los sujetos flotantes donde los referentes pierden su peso, los grandes dogmas se diluyen y la indeterminación alcanza su clímax con la desorganización, la flexibilidad de las normas y la inercia del espacio-tiempo, etc. Dibuja una sociedad que sufre de adolescentismo, inmadurez e incertidumbre.

Los flujos relacionales se construyen a partir de prácticas del individuo al espacio cotidiano, de modo que la comprensión del contexto, los códigos de conducta y otras dinámicas de socialización repercuten en la configuración de identidades (Adler-Nissen, 2014). En este proceso, los adolescentes construyen su lugar en el mundo a partir de sus percepciones. Navarro et al. (2015) alinean cuatro ejes influyentes en la configuración de la socialización de los nuevos perfiles de adolescentes: su autoconcepto a partir de las prácticas asociativas que establecen con el entorno, el imaginario colectivo del que participan, los modelos de representación social que construyen y, finalmente, su afinidad para/con éstos. 
Dichas dinámicas de asociación en y con el entorno conforman su identificación cultural con la sociedad donde viven y que construyen a imagen y semejanza de sus experiencias con iguales, familia, ámbitos curriculares formales y no formales y espacios de ocio (Lannes-Fernandes, 2012).

El ADN de la socialización de los adolescentes se configura a partir de las actividades cotidianas (Olszewski-Kuwilius et al., 2014); es decir, de la forma de resolver los conflictos, del cumplimiento de las normas, de las altas o bajas capacidades para afrontar los problemas, de la determinación en la toma de decisiones, de la tolerancia al fracaso, del afrontamiento ante nuevos retos, de la capacidad para retrasar las gratificaciones, de la asunción de responsabilidades...; como decimos, prácticas habituales. La nueva conciencia colectiva perturba las relaciones, dinamita el control social, incentiva el consumo y consiente los riesgos.

Las dificultades que encierra la adolescencia se propagan cuando los jóvenes son atraídos por dinámicas que integran riesgos (Rodríguez-Costas, 1999; Cuervo y Villanueva, 2013). Éstos se multiplican cuando además son compartidos por sus grupos de amigos. Las redes virtuales incrementan exponencialmente las dinámicas de riesgo, pues la audiencia a la cual se dirigen es ilimitada (Lareki et al., 2017). Algunos estudios, informan que la ruptura con los comportamientos integrados se produce a partir de la socialización del adolescente con entornos y prácticas de riesgo (San Juan et al., 2009; Son et al., 2014; Navarro-Pérez y Pastor-Seller, 2017).

El hecho ilícito de robar un coche o entrar en una propiedad privada son comportamientos delictivos que reproduce la criminología clásica y que necesitan de un ecosistema antisocial y necesidades criminógenas para su realización (Lamo de Espinosa, 1993; Moffitt, 1993). Sin embargo, las nuevas formas de asociación con el delito se configuran en un contexto cotidiano físico o virtual -violencia de género, suplantación de identidades, ciberacoso, bullying-; es decir, espacios normativos y prácticas (Wadesango y Machingambi, 2011), donde los adolescentes se socializan como víctimas, como agresores o como público.

En este sentido, Villanueva et al. (2008) refieren la audiencia como fenómeno diferenciador entre los comportamientos criminales clásicos y los actuales proyectados en la sociedad global; actualmente, las conductas reprochables son admitidas en contextos poblados, a la luz del día, en espacios habitados, dada la indiferencia que la comisión delictiva reproduce en la individualización del ser humano (Kenny, 2010) desafectado de conciencia social. Por tanto, las relaciones que se establecen en el marco de la adolescencia influirán en la sumisión a elementos de control social. 
En esta nueva era los adolescentes han sido atraídos por los encantos que envuelven las prácticas recreativas virtuales: redes sociales, juegos online y nuevas formas que permiten la autodeterminación. Morán (2002) considera que este perfil de adolescentes se encuentra sujeto a riesgos no perceptibles en las actividades cotidianas. Sin embargo, Tarín y Navarro (2006: 74) profundizan más en este análisis y etiquetan a estos adolescentes como "paralizados por el bienestar". Por su parte, Navarro-Pérez et al. (2016) los identifican como adolescentes con rasgos delictivos, pero con perfil de ajuste social; es decir, adolescentes que a priori se hallarían en contextos de protección, pero que a posteriori sucumbirían a las dinámicas de riesgo.

Son adolescentes "con rasgos prosociales y diferentes biografías, que acaban sometidos a la acción de la justicia" (Navarro-Pérez y Pastor-Seller, 2017: 20). Sugestionados por las inercias de la sociedad del riesgo (Beck, 1992), estiman tener cubiertas sus necesidades de integración social; no obstante se ven desbordados por el riesgo inapreciable que integran sus dinámicas cotidianas (Vega et al., 2012).

La literatura científica ha sido poco prolífica en tratar de revelar estos procesos de atracción al riesgo de adolescentes a priori integrados. Así pues, la delincuencia juvenil se ha explicado casi en exclusiva atendiendo a factores de riesgo estáticos, como la historia de vida familiar, o las experiencias o acontecimientos traumáticos en la infancia con disonancias en la adolescencia (Bowlby, 1985; Hayden et al., 2007). Sin embargo, en el estudio que aquí se presenta los riesgos acechan a los adolescentes desde cualquier actividad de la vida diaria.

García-Muñoz y Romero (2012) ofrecen una dimensión extensiva de los riesgos que colabora en la diversificación de los mismos y que afecta a los adolescentes en general, sin considerar las variables individuales de protección. En este sentido, De Lisi y Piquero (2011) señalan que la línea divisoria entre comportamientos prosociales y antisociales se ha visto reducida. En esta tesitura, Ryan et al. (2014) informan que las actividades ilícitas protagonizadas por adolescentes tienen alta asociación con las dinámicas cotidianas, ya que los comportamientos disruptivos pueden integrarse en ellas y, además, carecen del reproche público de tiempos pretéritos.

Por tanto, como decíamos al principio de la introducción, tanto el crecimiento o las posiciones acomodadas como el desarrollo social, educativo, tecnológico, etcétera, se encontrarían en el área de acción de las dinámicas de riesgo. 


\section{El sistema de justicia juvenil en España}

La entrada en vigor de la Ley Orgánica de Responsabilidad Penal de los Menores 5/2000 (LORPM, 2000) actualizó el escenario jurídico de atención penal a los adolescentes mayores de 14 y menores de 18 años. Un articulado basado en los modelos de justicia responsable que actualmente promueven las democracias occidentales y los estados progresistas (Jiménez, 2015). Un modelo que se construye a caballo entre la punición y la educación, con el principio de resocialización e interés superior del menor como baluartes principales.

Tres ideas principales guían la elaboración y puesta en marcha de la LORPM 5/2000: la responsabilidad penal, el superior interés del menor y la naturaleza sancionadora-educativa (Colás, 2011). Este articulado establece un marco flexible y presenta un amplio catálogo de medidas judiciales; unas de cumplimiento en medio abierto y otras de internamiento, que implican la pérdida de libertades. Así pues, el presente artículo analiza una muestra de Adolescentes en Conflicto con la Ley (ACL) privados de la libertad. Se pretende ofrecer respuestas desde los estilos de socialización en conexión con los riesgos.

\section{Metodología}

Hemos desarrollado una investigación multimétodo, construida a partir de la yuxtaposición de distintos procedimientos empíricos. Se ha dispuesto de diferentes técnicas, con el propósito de alcanzar objetividad y de conseguir para cada caso una línea de investigación coherente. Este planteamiento permitió estructurar y procesar la información obtenida, además de evaluar todas las fases del trabajo de campo y conseguir información general (encuestas) y particular (relatos de vida).

Por tanto, se han utilizado técnicas cualitativas (observación documental, entrevistas, grupos de discusión y relatos de vida) y cuantitativas (encuesta). Con base en las necesidades detectadas y las demandas de los profesionales que intervienen con este perfil de ACL e integrados en las áreas de justicia juvenil, educación y servicios sociales, se trató de investigar la realidad desde el foco del conflicto, con la intención de mostrar resultados replicables (Cook et al., 2009). Esta metodología basada en evidencias permite, según Flores (2013: 265), "abrir controversias" que garantizan la heterogeneidad y dibujan la realidad desde distintos planos analíticos, triangulando el método científico (Denzin, 1977). 


\section{Objetivos y consentimiento ético}

Consideramos prioritario mostrar los objetivos del presente estudio:

- Analizar ACL con perfil de ajuste sometidos a medidas de internamiento, explicando sus procesos relacionales y la asociación con prácticas de riesgo.

- Relatar las dinámicas que influyen en la conversión de actividades cotidianas en prácticas de riesgo en adolescentes, a priori, prosociales.

- Presentar una visión convergente entre los ACL y los profesionales-expertos que les atienden, para explicar su visión de la realidad y la extensión de riesgos que integran las actuales sociedades desarrolladas en contacto con las adolescencias.

\section{Participantes y método}

El número de sujetos que configuraron la muestra se obtuvo de los centros de internamiento judicial de la región de Valencia. La extensión y población de la provincia permitió integrar garantías de objetividad y representatividad para la propuesta metodológica. Antes de iniciarse el procedimiento empírico, se informó a los sujetos de los fines de la investigación. Un 91,7\% aceptó participar previa firma de consentimiento ético y utilización de los resultados para fines de divulgación científica y de transferencia de conocimiento para el desarrollo humano.

No se obtuvo respuesta del 6,9\% y, por tanto, no pudieron introducirse en el registro. El 1,4\% renunció a participar. Así, el 8,3\% -atendiendo a distintos motivos- no configuró el tamaño muestral. Respecto a informantes clave y expertos, se seleccionaron por cupos proporcionales de género.

Longitudinalmente se analizaron los años de 2011 a 2015, extrayéndose un tamaño muestral de 183 ACL con perfil de ajuste social de 14 a 18 años por la comisión de hechos sancionables penalmente y e internados judicialmente por tiempo no inferior a nueve meses con sentencia firme. Durante el proceso de observación documental en fuentes secundarias tales como memorias, claves de registro y catálogos de diligencias preliminares procedentes de la Fiscalía y de los tres juzgados de Menores de la provincia de Valencia, se habilitó como fuente primaria para completar el proceso empírico una ficha en la que se pudieron incorporar variables sociodemográficas, educativas, socializadoras, territorio-geográficas, relativas al perfil delictivo, que permitieron sistematizar, correlacionar distintas variables, complementar los resultados y 
el análisis posterior. Estos datos se incorporaron desde los expedientes judiciales de los ACL.

Con el objetivo de conformar el tamaño muestral de los ACL con perfil de ajuste social, se encuestó a 80 técnicos y educadores que atienden adolescentes infractores en los distintos centros de la provincia de Valencia con, al menos, cinco años de experiencia. Se realizó una encuesta multialternativa ad hoc (Wimmer y Dominick, 1996), que contenía alrededor de 50 características de distinta índole para identificar los criterios definitorios de los ACL con perfil de ajuste y, atendiendo al cumplimiento concurrido de estos, su inclusión muestral.

Dicha encuesta se realizó con la intención de objetivar el proceso empírico y ofrecer criterios de inclusión previamente contrastados por informantes expertos. Un 78,7\% de los encuestados devolvió la encuesta cumplimentada, configurando el perfil de ajuste a partir de seis variables. Se establecieron los criterios de More (2011) para proponer como variables de inclusión aquellas que lograran superar porcentajes de adhesión superiores al 75\%. Ello nos permitió establecer un rango de repetición de respuesta entre un $94,3 \%$ y un $75,8 \%$, identificando las siguientes características, definitorias de ACL con perfil de ajuste social:

1. Nula historia familiar afectada por patrones delictivos $\left(94,3 \% \mathrm{de}_{\mathrm{TaR}}{ }^{1}\right)$.

2. Ausencia de subordinación a entidades caritativas, de beneficencia o intervención desde instituciones públicas de bienestar social (87,1\% TaR).

3. Núcleo familiar ubicado en territorios con amplia dotación (83,3\% TaR).

4. Amplia capacidad para la integración social/laboral del núcleo familiar y para la gestión relacional con redes prosociales de apoyo (80,1\% TaR).

5. Disponer de independencia económica para afrontar las necesidades sociofamiliares de ocio y consumo sin necesidad de terceros (77,8\% TaR).

6. Cumplimiento de sanción penal firme superior a nueve meses por la comisión de delitos distintos a la violencia filioparental o en concurso penal con éste (75,8\% TaR).

Adicionalmente se realizaron 20 entrevistas a distintos profesionales que atienden adolescentes para establecer la complementariedad de método. Los expertos entrevistados tenían experiencia superior a diez años desde distintas pero complementarias áreas: educativa, justicia penal y restaurativa, y servicios de bienestar y participación sociocomunitaria. Era fundamental, en este sentido, acercar la percepción de profesionales que interactúan con adolescentes, no solamente desde una perspectiva punitiva, sino a través de multienfoque.

1 Tasa de Respuesta. 
Las recomendaciones de Fonseca et al. (2017) proponen que en el proceso secuencial es imprescindible analizar el objeto desde una perspectiva heterogénea, contando con variada especialización de informantes para configurar un análisis construido en distintos parámetros de opinión. En la Tabla $1^{2}$ aparecen los expertos entrevistados. Previa y posteriormente se planificaron dos grupos de discusión; el primero con el fin de elaborar un guión de entrevista a los expertos, y el segundo (Grupo de Expertos) para verificar los resultados derivados del proceso de triangulación de técnicas.

Las entrevistas abordaron esencialmente indicadores del tipo socialización familiar, rutinas cotidianas, iguales, y planificación del tiempo libre, gestión de las dinámicas relacionales y de riesgo e inicio de las conductas delictivas. Asimismo, se efectuaron ocho relatos de vida de ACL con perfil de ajuste, para, desde esta percepción, explicar los riesgos de la era global y plasmar las experiencias vitales de los adolescentes en asociación con los riesgos, desde un formato autobiográfico con vocación reflexiva (Bezerra de Brito, 2013).

Una vez que los informantes claves expertos nos ofrecieron su visión genérico-global de los riesgos, nos interesaba poner el foco de atención en la percepción subjetiva de los propios adolescentes y valorar si ésta se alineaba con la panorámica de los expertos. De este modo conseguimos cerrar la secuencia objetiva y cercar el proceso empírico, siguiendo las fases de Cardona (2002), de lo general a lo particular. Los relatos trazaron una línea vital a partir de factores estáticos (de la historia familiar y personal e infancia de los sujetos) y posteriormente dinámicos, en virtud de procesos de socialización secundaria y terciaria.

La selección de informantes atendió a cuotas a partir de la experiencia en el trabajo con adolescentes, el sector de intervención y la procedencia institucional público/privada. La Tabla 1 muestra estas características junto con la codificación que les fue asignada. Los relatos de vida de la Tabla 2 presentan 8 de los 183 ACL del tamaño muestral.

El tratamiento cualitativo para la codificación discursiva tanto en entrevistas como en grupos de discusión y en los relatos biográfico-narrativos se realizó con software para el tratamiento de datos cualitativos MAXqda versión 12. La sistematización textual se organizó en tres agrupaciones: texto, concepto y significación discursiva. Se estructuraron distintas categorías iniciales, reducidas finalmente tras la verificación del segundo Grupo de Expertos a dos de ellas: dinámicas relacionales y riesgos hallados en éstas. Además,

2 Todas las tablas y las figuras se encuentran en el Anexo, al final del presente artículo (Nota del editor). 
utilizamos el programa Excel para sistematizar las variables demográficas representativas de la muestra.

\section{Resultados}

Presentamos, en primer lugar, las dinámicas relacionales de los ACL con perfil de ajuste, para después presentar los riesgos en los que cotidianamente se integran.

\section{Dinámicas relacionales o estilos de socialización}

a) Nuevas prácticas de asociación familiar

La comunicación entre padres e hijos se ha visto dañada por la aparición de dependencias afectivas, de salud, problemas emocionales, rupturas y nuevos vínculos de pareja...

Aquí ha habido padres que han venido a visitar a sus hijos y te preguntan: "Oye, tal, ¿y de qué hablo con mi hijo?”... ahí algo no funciona. (PSIER)

La institución familiar ha evolucionado en las formas de interactuar de sus miembros. Del padre autoritario se ha reconvertido en progenitor dialogante y flexible. La Figura 1 establece cuatro indicadores para representar el estado de estas relaciones tanto positivas como beligerantes entre padres e hijos. El 71\% mostró conflictividad con sus progenitores, el 16\%, relaciones inestables y sólo 1 de cada 10 ACL afirmó mantener relaciones positivas con ellos.

A los padres, cada vez les cuesta más ser normativos con sus hijos... y, a los adolescentes hay decirles, ahora no y por aquí, sí. (MIPE1)

Informantes entrevistados, expertos y ACL coincidieron en señalar la coherencia como la principal estrategia educativa. Tal vez una faceta que "no se enseña”, pero que se convierte en prioritaria cuando los hijos son difíciles.

La coherencia no se enseña, es algo innato... por eso es tan difícil... (OIPE)

Lo que más me rallaba de mi madre era que me mareara. Me decía una cosa y por lo mismo otro día, lo contrario... y yo ahí ya me flipaba y empezaban las trifulcas. (4)

Los padres tienen la exigencia moral de ejercer funciones de responsabilidad. La educación ha perdido ascendencia y representatividad. El respeto a las personas mayores que conviven en el núcleo familiar ha ido despojando los fundamentos de las relaciones de familia, donde el miembro mayor gozaba de la experiencia de la vida. 
¿Dónde están los abuelos?... es otra pérdida para que los chavales aprendan a respetar desde su ombligo. (TSSSB1)

Si los padres no son responsables, ¿̇cómo lo van a ser de sus hijos? (EMJMA1)

Hay chavales que piensan con más lucidez que sus padres... aquí en la Comisaría ves algunas situaciones que dices, normal que el crío haga lo que hace. (GRUME)

La comprensión y cercanía a los hijos han acabado por constituir interacciones horizontales donde padres y madres se acogen a un rol de amigo, antes que a un estilo directivo. La actividad educativa de los padres queda supeditada al escenario de las buenas relaciones y, en algunos casos, a la manipulación. La profunda reflexión de las autobiografías así lo indicaron:

...dos años encerrado por las movidas... Me creía el puto King. (6)

Mi madre no es mi madre, o sea, sí... pero en verdad es más amiga que madre. (5)

A mi madre con dos carantoñas me la camelaba. (3)

\section{b) Consumismo desmedido}

El bienestar actual con el que se goza de amplias condiciones de calidad de vida y favorece el desarrollo humano se posiciona paradójicamente contradictorio al valor del esfuerzo, del trabajo para conseguir bienes de consumo y de última generación.

Me he comprado ropa en el Zara que ni me he puesto. (1)

En general, la gente valora más una idea brillante que el esfuerzo de toda una vida..., el que inventó Facebook..., una idea y a vivir de rentas. (PIPE)

La Figura 2 presenta referencias de consumo con alta ascendencia para los ACL con perfil de ajuste. Podemos observar cómo las nuevas tecnologías han colapsado los intereses de los ACL; un 65\% refiere el smartphone como bien de interés destacado en su escala de prioridades, por delante de otros como el capital efectivo (18\%). La ropa y los complementos continúan siendo bienes con ascendencia, seguidos de la devoción por tener cuerpos modélicos, tatuajes, cirugías u otros modelos de belleza (5\%).

Sin mi iPhone no voy a ningún sitio. (4)

Si no tienes whatsapp, estás en otro planeta... y si eres adolescente, en otra galaxia... (TSSSE)

La familia ha evolucionado también en lo que a roles se refiere. Antes trabajaba el padre, y la madre estaba dedicada a las tareas del hogar. Ahora la mujer se ha incorporado al trabajo, rompiendo roles patriarcales y favore- 
ciendo un mayor capital económico familiar, lo cual implica más capacidad adquisitiva y un impulso del consumo en los hogares.

Las familias por lo general tienen más posibilidades de capitalizarse, pero el ahorro es nulo. O sea, como los chavales, dinero que entra... dinero que funden. (OIPE)

Los centros comerciales son el opio del pueblo del siglo XXI. Los hogareños estamos en peligro de extinción. (PSSB2)

La tecnología y los avances científicos albergan nuevos logros. El placer de comprar nuevas versiones constituye una forma de vida. La globalización actual se caracteriza por sobreproducir. La cultura de la abundancia inunda los hogares, poniendo su foco en las nuevas generaciones para que reparar o reutilizar pasen a ser conceptos en desuso.

Hay que diferenciar entre la globalización y la globalización 4.0. Es más rentable comprar una cosa nueva que arreglarla. (ESIAI)

Es más barato comprarme una plancha del pelo que arreglarla. (3)

\section{Riesgos que acompañan al cambio social}

a) Valores denostados

El efecto de alcanzar los objetivos de manera rápida y poco elaborada hace que la inmediatez suponga un importante refuerzo. Una sociedad avanzada y ultra estimulada.

La demora de las gratificaciones es una tarea que procede de la rica sabiduría oriental.

Lo de esperar la oportunidad y aprovecharla está desfasado. (MIPE2)

Adultos y adolescentes hemos hallado confort en estas rutinas de inmediatez. Por tanto, el corto plazo constituye un nuevo estilo de vida.

Lo de "lo quiero para ayer" es una forma habitual de pedir las cosas. (PSIER)

El futuro no existe..., dame lo que tengas hoy que a lo mejor mañana no llega nunca. (5)

La educación de padres a hijos está cubierta de distintos resortes que facilitan los deseos de los adolescentes. De este modo, si ponemos a estos en una urna, nunca serán capaces por sí mismos de tolerar frustraciones y gestionar adecuadamente sus decepciones.

Es bueno que los padres dejen unos saquitos de carbón la noche de Reyes..., que los hijos mejoren, es tarea de los padres. No podemos resolver todo con premios. (PETJ)

De pequeña he tenido todo lo que he querido; me portara bien o mal. (7) 
b) Disfuncionalidad de las actividades recreativas

La ausencia de una planificación cotidiana implica dinámicas de alto riesgo. Por tanto, es de sumo interés que los adolescentes dispongan de una red de recursos y actividades prosociales completa. Además, es importante el compromiso tanto de los padres como de las instituciones públicas por ofrecer actividades y políticas de ocio que garanticen el acompañamiento, la participación y la creatividad de los adolescentes. Las nuevas tecnologías envuelven la recreación de los adolescentes.

Si eliminas los clubs de futbol, los grupos scouts y los Juniors o los grupos de Fe de la

Iglesia católica, dime qué te queda para que los chavales se diviertan. (MTIER)

Compartir un puzzle suponía una pericia familiar..., los videojuegos, la play..., se ha cargado estos momentos. (MIPE1)

Habría que considerar a las incesantes críticas al botellón ${ }^{3}$ desde una perspectiva institucional, planteando las opciones que se ofertan a los adolescentes para evitar estas rutinas de riesgo, a todas luces contrarias al fomento de hábitos positivos.

El problema es que no hay regulación y que además pasa los fines de semana, que es cuando los muchachos se liberan de sus obligaciones. (OIPE)

Ponerse to morao, a ver quién bebe más y quien se le va más la cabeza..., eso es para mí, el botellón. (2)

En la Figura 3 se indica que casi 7 de cada 10 ACL (68\%) no participó en actividades de ocio prosocial. Tan sólo el 17\% lo hizo de manera regular y el $11 \%$, intermitentemente. Recordemos que uno de los criterios de inclusión muestral fue la existencia de recursos prosociales en sus entornos de residencia; es decir, las posibilidades de establecer dinámicas relacionales positivas es favorable, aunque es obvio que las actividades que se ofertan no interesan a los ACL.

¿Lo que hacía en mi ocio? Pues ná. Comer pipas, fumar marihuana..., y de vez en cuando "de palo $\mathrm{y}^{4}$ de putas". (8)

Si queremos que los adolescentes se monten su oferta de ocio, tendremos que prepararnos para que hagan algo que no nos guste. (EPSSB)

3 Actividad realizada por los adolescentes y que consiste en beber alcohol en la vía pública durante los espacios recreativos especialmente nocturnos, con la intención de pasar el rato, escuchar música, hablar y, en algunos casos, bailar.

4 Cometer un delito en grupo. 
Los juegos constructivos, educativos, colectivos han sido casi extinguidos por otros online, los videojuegos o las aplicaciones de telefonía móvil que permiten en una sola descarga la adquisición de paquetes de entretenimiento. Se promueve una recreación que tiende a individualizar a los sujetos o dinamizarlos en redes virtuales, sin contacto físico. Los juegos han perdido el acento de sagacidad, promotor del pensamiento creativo.

Los videojuegos siembran personalidades autómatas. Tienes un objetivo, conseguir esto..., pues a matar naves espaciales o a pasar pantallas como un robot. (TSSSB1)

Para divertirte es mejor no pensar..., si piensas, no te diviertes. (4)

Todo forma parte de una macrooperación..., pensar poco. Criamos adolescentes sin conciencia crítica. (PIPE)

En este sentido, la confusión que generan nuevos estímulos, adentrarse en nuevas formas lúdicas como las partidas de póker online o la nueva esfera de las apuestas, enmascara las dinámicas de ocio e impulsa un tobogán de riesgos. En el siguiente apartado ampliaremos esto.

Empecé tonteando con las apuestas y al final tenía que robar para jugar. Son una droga, bueno peor..., porque con la cocaína si no tienes no consumes, pero para las apuestas sólo necesitas Wi-Fi. (6)

¿Las apuestas? Una locura..., suplantan la identidad de su padre y a jugar. (ESIER)

c) La naturalización del consumo de drogas: nuevas y viejas dependencias

Tradicionalmente, las drogas han sido temidas por su seducción y posterior dependencia. El sometimiento a ellas está motivado por lo que los informantes explican como "condicionantes de fragilidad"; es decir, las circunstancias adversas que rodean al individuo y lo hacen vulnerable; quebrantable a las drogas, al sexo, a las partidas de póker, a las apuestas u otras situaciones que ponen en peligro la voluntad subjetiva.

Hay condicionantes que les llamo de fragilidad que esclavizan a un hábito..., y pasa porque el adolescente es frágil por la naturaleza que le rodea, y no por si fuma más o menos hierba o tiene cuentas en una o cien casas de apuestas. (EDETJ1)

Tengo amigas que nunca se han drogao... Dicen que nos drogamos por inseguridad. (1)

El acceso natural a la droga es una rutina que paulatinamente se convierte en práctica habitual. De ahí la importancia que estos condicionantes de fragilidad no socaven la percepción del adolescente sobre los comportamientos que naturaliza. 
No es lo mismo fumarse un porro de marihuana que esnifar una raya de cocaína un martes después de almorzar. (MTIER)

A nadie le extrañaría ver a un chico de 16 años de cervezas y fumando un cigarrillo en la calle, pero sí que esnifase speed. A eso me refiero, que el chaval tiene que saber cuándo y dónde puede hacer una cosa y cuándo y dónde podría hacer otra. (TSSSE)

En la muestra de ACL analizados, un porcentaje ínfimo (12\%) no reflejó patrones de consumo dependientes, frente a un $88 \%$ que representó atractivo. En la Figura 4 aparecen los $161 \mathrm{ACL}$ con perfil de ajuste en quienes se halló receptividad hacia el uso/abuso de drogas. Observamos cómo el policonsumo de sustancias con un $93 \%$ se eleva como referencia, seguido de las redes sociales virtuales con un $87 \%$, la marihuana como derivado cannabinoide con un $85 \%$, las apuestas deportivas con un $72 \%$ de los casos por delante del tabaco con $71 \%$ y alcohol con el $66 \%$.

Destacamos también el resurgimiento de los opiáceos (heroína) con el $23 \%$, casi al mismo nivel que estimulantes como la cocaína (26\%). Es significativa la mayor receptividad a la marihuana que al tabaco, aunque para su consumo, habitualmente, se utilizan ambas sustancias. No obstante esta representación, queda como principal referencia el elevado impacto que las nuevas dependencias provocan en este perfil de ACL.

El caballo vuelve por sus fueros..., pero la droga del futuro son las apuestas y el póker por Internet. (ESIAI)

Farlopa, maria, keta.... póker, apuestas, sexo y putas... Hay de todo, como en un mercadillo de esos, que te compras unas bragas y un kilo de cebollas. (6)

En muchas ocasiones, los adolescentes pierden la dinámica estructural prosocial en poco tiempo, asumiendo rutinas de riesgo que les hacen olvidar sus actividades integradas.

En un verano loco..., de sacar buenas notas a dormir en la calle. (MEIPE)

Antes de entrar al centro, algunos se fumaban 20 canutos, con speed, durmiendo poco, robando mucho... Eso a diario es un coctel que es difícil restituir. (EMJMA1)

Las dinámicas disruptivas hallan su cultivo en las dependencias y en la flexibilidad e incertidumbre del entorno, que favorece el mantenimiento de prácticas de riesgo.

d) El contexto físico / virtual: potencialidades y riesgos

El contexto en el cual se relacionan los adolescentes ha evolucionado; hemos pasado de una esfera física, donde la calle ejercía una influencia preponderante, 
a espacios virtuales en los que es muy sencillo esconderse o manipular la imagen que se pretende transmitir.

En la calle no hay youtubers o plataformas de descarga. Por eso, su interés está en otros sitios. (MEIPE)

Tengo tres o cuatro perfiles de Facebook..., igual que mis amigos. (2)

La socialización virtual provoca que el diálogo físico pierda referencia. Los problemas interpersonales se resuelven en muchos casos con decisiones unilaterales. Pero, por otro lado, el espacio virtual constituye una auténtica red de relaciones. La audiencia de Internet es ilimitada y los estímulos atienden al número de likes.

Si me ralla, le cierro la boca del chat..., sin explicar nada. No hace falta. (7)

¿Cuantos "me gustas" tienes?... Así funciona ahora la vida. (ESSSB)

En Internet, el territorio local queda subordinado a una esfera más amplia marcada por lo global. La inmediatez de la esfera virtual no se corresponde con la pausa, la espera o los valores de la escena física. Las expectativas de la escena física confrontan con las realidades inmediatas del recreo virtual.

Hay que enseñarles a separar los dos mundos, aunque para ellos sea uno solo. (FIJ)

Estos chavales.... Twitter o Instagram es lo que a Moisés la tierra prometida. (ESIAI)

\section{Discusión}

El objetivo en sentido amplio de este trabajo fue acercarnos a la realidad de los nuevos perfiles de ACL, en concreto a los que tienen perfil de ajuste social. Para ello, nos servimos de la visión de profesionales expertos y de las experiencias de los propios adolescentes, con el fin de explicar las dinámicas relacionales y que directa o inversamente afectan su desarrollo prosocial y sus dinámicas cotidianas en contacto con el riesgo. Así, trazaremos la discusión apoyada en literatura científica que permita canalizar los análisis expuestos.

\section{Autodeterminación, nuevos conceptos, erosión familiar y trivialidad de valores}

La familia se ha erosionado en las últimas décadas (Aguiar, 2015). Hemos visto en los resultados cómo la estructura familiar ha adquirido nuevos registros. La cultura, los nuevos roles de padres e hijos, la confusión sobre los modelos de comportamiento intrafamiliar (Olszewski-Kuwilius et al., 2014) reproducen nuevas pautas de convivencia. 
En esta línea, Meil-Landwerlin (2011) refiere que el desvanecimiento del patriarcado y los nuevos roles influyen en estos cambios. Así pues, la familia ejerce menor influencia en los adolescentes; además, los padres también han caído en la seducción de las nuevas formas de vida. En el hogar, no sólo los adolescentes exigen su espacio de independencia, sino que los padres también necesitan libertad para reconfigurar su vida cotidiana. Esto afecta la idiosincrasia de familia como grupo y a la comunicación entre los miembros. Las actividades recreativas que antaño unían se han reconvertido en prácticas individuales. Los intereses particulares han fracturado la vida en común (Pérez-Agote, 2010). En este contexto, el todo no equivale a la suma de las partes, estableciéndose ausencias destacadas que durante la adolescencia condicionan el desarrollo prosocial.

La era del cambio social es fruto del progreso tecnológico, científico, del desarrollo de las telecomunicaciones, pero también de nuevos escenarios para el conflicto. En los resultados hemos analizado cómo el consumismo ferviente, la pérdida de valores o las nuevas dinámicas de gestión familiar han comprometido la prosperidad de los nuevos tiempos. Los adolescentes utilizan las tecnologías para autodeterminarse. Los grupos de whatsapp o las cuentas en Facebook permiten una socialización paralela a la de sus padres, en la línea de las transgresiones identitarias de Adler-Nissen (2014).

Coincidimos con Lee y Lee (2017), en que el Smartphone introduce nuevos usos tanto en la relación de los adolescentes con su entorno, como en la primacía que por sí mismo representa. Los bienes materiales se han disparado por encima de otros etéreos hasta ahora indiscutibles como el esfuerzo, el trabajo, la responsabilidad, el diálogo, la tolerancia... La evolución social ha depreciado los valores e incluso pone en tela de juicio su vigencia a los ojos de las nuevas generaciones. Por tanto, nuevos conceptos inundan nuestro diccionario cotidiano: actualización, desconfigurado, vida útil, desuso... Estas son palabras con las que las nuevas generaciones continuamente entran en relación.

En esta línea, Comba et al. (2012) informan que aquello que carece de operatividad pierde su valor y pronto queda desactivado. Así, la histeria de las actualizaciones, los riesgos de la evolución tecnológica desmedida condicionan lo que tienen y, en consecuencia, lo que son (representan) los adolescentes.

Los mecanismos de control social y las instituciones reguladoras de la actividad humana, como ha venido siendo la familia, siguen existiendo; sin embargo, los procesos de subjetivación determinan los intereses individuales frente a la vida comunitaria. En este análisis coincidimos con OlszewskiKuwilius et al. (2014) al desarrollar la idea de que la calidad de vida y los 
postulados de autorrealización personal legitiman la felicidad frente a la seguridad económica, el ahorro o la posesión patrimonial de tiempos pretéritos. Esta evolución es positiva porque favorece la soberanía de los miembros, pero, por otra parte, fragmenta la solidez del núcleo.

Los resultados mostraron adversidades de las adolescencias y cómo la evolución social ha influido en su atracción al riesgo. La adolescencia es energía, pujanza, vitalidad, pero también es inexperiencia, incerteza, búsqueda. Shifflet-Chila et al. (2016) refieren que las grandes instituciones como la familia deben impulsar el tránsito hacia la madurez. En este sentido, es vital atender a los planteamientos de Sündermann et al. (2014) que recurren a la conciencia creativa y crítica como factores de protección, para que los adolescentes configuren sus procesos de desarrollo ajenos a las modas e incrementando el pensamiento propio, alejado del bullicio consumista, factor que previene el contagio de los riesgos.

\section{Riesgos en la realidad social (virtual)}

La cuestión de fondo que se plantea en este artículo es la siguiente: ¿estamos todos sometidos al riesgo de las nuevas sociedades desarrolladas? Efectivamente. Daset et al. (2007) coinciden con nuestros hallazgos en que las dinámicas de riesgo son prácticamente inapreciables, porque el bienestar colectivo y el desarrollo científico técnico las enmascara. Los actuales adolescentes han nacido en un periodo histórico pródigo (Brener y Schmid, 2016). No son conocedores del hambre, ni de la guerra ni del miedo. Criados en la burbuja del resplandor. En esta esquizofrenia, los adultos intentan alargar la juventud (Suárez-Bautista, 2009), y los adolescentes pretenden ser adultos de una sola vez. Es el mundo al revés. Invertir la lógica de la evolución es audaz pero viable a causa de la indefinición de los valores y el mutismo de instituciones sacras.

La ascendencia que el territorio físico y la red de recursos tiene para los adolescentes nos alerta sobre una despreocupación de la esfera "real", en detrimento del vasto conocimiento que posee el universo virtual. Los adolescentes que aquí se describen no participaron en un alto porcentaje (68\%) de actividades recreativas organizadas, pese a que disponían de tiempo libre para llevarlas a cabo. El contexto físico pierde su importancia y pasa a formar parte de una realidad virtual con elevada ascendencia, en la cual encuentran más estímulos pero también, como hemos descrito, más dependencias. Existe una desafección en la participación de la vida social de estos ACL, coincidiendo con el estudio de De la Torre y Baquerín de Riccitelli (2017) con jóvenes argentinos. 
Nuestros hallazgos son análogos a investigaciones que diagnostican un ocio individualizado no planificado (Lannes-Fernandes, 2012), con escasa representatividad grupal y únicamente latente en deportes colectivos (Murzi y Czesli, 2015) y en atracciones de recreación nocturna (Measham y Hadfield, 2009). Por último y compartiendo nuestro análisis, Huang et al. (2010) advierten del incremento de la adicción de los adolescentes a juegos online; una recreación tecnológica que en sí misma evidencia riesgos (Young, 2007) y que muestra escasa funcionalidad para promover el espíritu crítico, convirtiendo el juego y las prácticas de entretenimiento en entes vacíos de inspiración e inventiva.

Nuestros hallazgos coinciden con las aportaciones de Navarro et al. (2016), en las que identifican la heterogeneidad del actual perfil criminal del adolescente; es decir, una descripción que no sólo se asocia a problemáticas de marginación y exclusión social, sino que está determinada por la conexión o tiempo de exposición a los riesgos que entrañan las nuevas sociedades y que, en muchos casos, según Berga (2011), son invisibles en la socialización cotidiana.

\section{Conclusiones y propuestas}

Los ACL que hemos presentado son consecuencia directa del desarrollo con escasa atención a los valores, a la tradición y a las normas. Son resultado de los excesos. Exceso de flexibilidad, de modernidad, de libertades, de la democratización de instituciones como la familia, de la elasticidad del nuevo orden social, del comercio de las relaciones humanas, de un mundo frustrado y obnubilado con los avances y el placer subjetivo. En definitiva. Los adultos hemos creado un escenario de comodidad, provocando una derivada negativa en nuestro contexto diario que condiciona la vida cotidiana, especialmente de aquellos más vulnerables entre quienes se encuentran los adolescentes.

El presente artículo nos ha hecho reflexionar en que el riesgo no entiende de clases ni de posiciones o condiciones sociales, y que se adentra a medida que las vulnerabilidades aumentan. La sociedad es de riesgo y éste se impregna en la socialización de los seres humanos. Otra de las cuestiones de sumo interés que nos deja este trabajo es que el riesgo es global y que las adolescencias socializan en torno a él. Queda también para la reflexión la idea de que los riesgos de adultos y adolescentes son los mismos; por tanto, habrá que cuestionarse: ¿cómo estamos protegiendo a las futuras generaciones?, ¿en qué medida estamos impulsando que asuman pautas de comportamiento adulto? o ¿cómo les ayudamos a identificar y evitar los icebergs de riesgo que nos rodean? 
Es prioritario que invitemos a los adolescentes a pensar por sí mismos, a evitar modelos de copia y alejarse de los pseudoavances. Los adultos hemos de reconfigurar nuestro entorno para ofrecer nuestra mejor versión a las futuras generaciones. En esta vorágine difusa de incertidumbres y progresos planteamos algunos aspectos para apoyar al adolescente ante su contacto con el riesgo:

1. Fortalecer la dimensión colectiva de la participación y la recuperación del territorio físico, de la calle, para promover dinámicas asociativas y participativas en grupos. Establecer relaciones armónicas entre el contexto físico y el virtual.

2. Acompañar las adolescencias desde una mirada adulta, recuperando los valores de proximidad, favoreciendo la reflexión, la coherencia y el diálogo.

3. Cuestionar los estereotipos generalistas que buscan la imitación como estilo y forma de vida. Promover estas acciones desde el currículum formal en asignaturas transversales orientadas al alumnado de todas las edades.

4. Promocionar políticas de inversión en valores sociales y pensamiento crítico a todos los niveles, no sólo en prevención especial, sino, lo que es más importante, desde la prevención general.

5. Apostar por el patrimonio cultural y la recuperación de bienes ilustrativos de una ciudadanía libre, responsable, solidaria y tolerante.

6. Proyectar el futuro sin perder la vista el pasado, porque los aprendizajes, errores y experiencias ayudarán a la evolución social y al progreso humano.

La calidad de vida integra un pensamiento equívoco de perenne bienestar; por tanto, es necesaria una reflexión que contribuya al crecimiento colectivo futuro, tanto de adolescentes en general, como de ACL con perfil de ajuste en particular.

\section{Referencias}

Adler-Nissen, Rebecca (2014), "Stigma management in international relations: transgressive identities, norms, and order in international society”, en International Organization, vol. 68, núm. 1, Reino Unido: Cambridge University Press.

Aguiar, Francisco (2015), "Solidaridad intergeneracional de las familias: abuelas y abuelos en el cuidado de la infancia”, en Revista de Servicios Sociales y Politica Social, vol. 107, España: Consejo General de Asistentes Sociales y Diplomados en Trabajo Social.

Beck, Ulrich (1992), Risk Society: Towards a New Modernity, Inglaterra: Sage.

Benavides, Abraham (2013), “Tour major disaster occurences and the Spanish lenguaje media: a lack of risk communication”, en Disaster Prevention \& Management, vol. 22, núm. 1, Reino Unido: Emerald Group Publishing. 
Convergencia Revista de Ciencias Sociales, núm. 76, 2018, Universidad Autónoma del Estado de México

Berga, Anna (2011), "Chicas adolescentes y conductas transgresoras: entre el riesgo y la invisibilidad”, en Oriol, Romarí y Planas, Anna [coords.], Jóvenes y riesgos: ¿unas relaciones ineludibles?, España: Bellaterra.

Bezerra de Brito, Daniel (2013), "Práctica pedagógica, saberes e historias de vida”, en La Recherche en Éducation, núm. 10, Francia: Institute Nationale de Recherche Pedagogique.

Bowlby, John (1985), La separación afectiva, España: Paidós.

Berger, Peter y Huntington, Samuel (2002), Globalización múltiple. La diversidad cultural en el mundo contemporáneo, España: Paidós.

Brener, Neil y Schmid, Christian (2016), “La 'era urbana' en debate”, en URE: Revista Latinoamericana de Estudios Urbano Regionales, núm. 127, Chile: EURE.

Cardona, María (2002), Introducción a los métodos de investigación en educación, España: EOS.

Colás, Asunción (2011), Derecho Penal de Menores, España: Tirant lo Blanch.

Comba, Silvana et al. (2012), "Algunas consideraciones sobre las prácticas de comunicación, consumo y producción cultural en los nuevos medios en jóvenes rosarinos”, en Austral Comunicación, vol. 1, núm. 1, Argentina: Universidad de Austral.

Cook, Bryan G. et al. (2009), "Determining evidence-based practices in special education", en Exceptional Children, vol.75, núm. 3, Estados Unidos: Council for Excepcional Children.

Cuervo, Karen y Villanueva, Lidón (2013), "Reiteración y reincidencia delictiva en menores españoles con expediente judicial”, en Revista Mexicana de Psicología, vol. 30, México: Sociedad Mexicana de Psicología.

Daset, Lilián et al. (2007), "Valores Humanos Básicos en un grupo de jóvenes: avance de un estudio exploratorio”, en Psicodebate. Psicologia, Cultura y Sociedad, vol. 7, Argentina: Universidad de Palermo.

De Lisi, Monhan y Piquero, Alex (2011), "New frontiers in criminal careers research, 2000 - 2011: A state - of - thre - art review”, en Journal of Criminal Justice, vol. 39, núm. 4, Reino Unido: Elsevier.

De la Torre, Lidia y Baquerín de Riccitelli, María (2017), "Los jóvenes argentinos que no estudian ni trabajan; déficit de integración social”, en Reis: Revista Española de Investigaciones Sociológicas, núm. 158, España: Centro de Investigaciones Sociológicas.

Denzin, Norman (1977), The research act: a theoretical introduction to sociological methods, Inglaterra: Prentice Hall.

Flores, Pedro (2013), "El enfoque de la política basado en la evidencia: Análisis de su utilidad para la educación de México”, en Revista Mexicana de Investigación Educativa, vol. 18, núm. 56, México: Consejo Mexicano de Investigación.

Fonseca, Eduardo et al. (2017), "Effects of an Emotional Education Intervention in Students of University Programs for Older Students", en Universitas Psychologica, vol. 16, núm. 1, Colombia: Pontificia Universidad Javeriana. Disponible en: http://dx.doi. org/10.11144/Javeriana.upsy16-1.eiee [4 de marzo de 2017].

Galbraith, John Kenneth (1997), La sociedad opulenta, España: Ariel.

García-Muñoz, María y Romero, César (2012), "Nuestros, vuestros y suyos. Perfil de los adolescentes en conflicto con la ley en Burjassot", en Revista de la Asociación de Sociología de la Educación-RASE, vol. 5, núm. 2, España: Asociación de Sociología de la Educación.

Hayden, Carol et al. (2007), "Schools, pupil behaviour and young offenders: Using postcode classification to target behaviour support and crime prevention programmes", en British Journal of Criminology, núm. 47, Reino Unido: Oxford University Press.

Huang, Xui-Qui et al. (2010), “Treatment of internet addiction”, en Current Psychiatry Reports, vol. 12, núm. 5, Estados Unidos: Current Science. 
José Javier Navarro-Pérez y Enrique Pastor-Seller. De los riesgos en la socialización global: adolescentes en conflicto con la ley con perfil de ajuste social

Jiménez, María (2015), "Algunas reflexiones sobre la responsabilidad penal de los menores", en Revista Electrónica de Ciencia Penal y Criminología, vol. 17. España: Instituto Andaluz Interuniversitario de Criminología.

Kenny, Robert (2010), "Beyond the Elementary Forms of Moral Life: Reflexivity and Rationality in Durkheim's Moral Theory”, en Sociological Theory, vol. 28, núm. 2, Estado Unidos: Sage.

Lamo de Espinosa, Emilio (1993), Delitos sin víctima. Orden social y ambivalencia moral, España: Alianza.

Lannes-Fernandes, Fernando (2012), “Os jovens da favela. Reflexões sobre controle e contenção sócio-espacial dos párias urbanos no Rio de Janeiro”, en Convergencia. Revista de Ciencias Sociales, vol. 59, México: Universidad Autónoma del Estado de México.

Lareki, Arkaitz et al. (2017), “Teenagers perception of risk behaviors regarding digital technologies", en Computers in Human Behavior, vol. 68, Reino Unido: Elesevier.

Lee, Changho y Lee, Sook-Jung (2017), "Prevalence and predictors of smartphone addiction proneness among Korean adolescents”, en Children \& Youth Services Review, vol. 77, Reino Unido: Elsevier.

Lipovetsky, Gilles (2007), La era del vacio: ensayos sobre el individualismo contemporáneo, España: Anagrama.

LORPM (2000), "Ley Orgánica de Responsabilidad Penal de los Menores 5/2000”, de 12 de enero, en Boletín Oficial del Estado, núm. 11, España. Disponible en: https://www.boe. es/boe/dias/2000/01/13/pdfs/A01422-01441.pdf [24 de octubre de 2017].

Measham, Fiona y Hadfield, Phil (2009), “Todo empieza con 'E': exclusión, etnicidad y formación de élites en el mundo actual de las discotecas inglesas”, en Adicciones: Revista de socidrogalcohol, vol. 21, núm. 4, España: Asociación Socidrogalcohol.

Meil-Landwerlin, Gerardo (2011), Individualización y Solidaridad Familiar, España: Fundación La Caixa.

Moffitt, Terrie (1993), "Life Course Persistent and Adolescence Limited Antisocial Behavior", en Psycological Review, núm. 100, Estados Unidos: American Psychological Association.

Morán, José (2002), Delincuencia juvenil en la clase media, España: Promociones y Publicaciones Universitarias.

More, Chandramouli (2011), "The effect of Appreciative Inquiry as organizational development intervention on organizational planning and service quality improvement in St. Francis School (ICSE)", en Revista de Cercetare si Interventie Sociala, vol. 33, Rumania: Experts Projects Publishing.

Murzi, Diego y Czesli, Federico (2015), "Apuntes sobre una intervención para deconstruir las condiciones que generan la violencia en el fútbol”, en Lúdica pedagógica, vol. 2, núm. 21, Colombia: Universidad Pedagógica Nacional.

Navarro, José Javier et al. (2015), “El proceso de socialización de los adolescentes postmodernos. Entre la inclusión y el riesgo: recomendaciones para una ciudadanía sostenible”, en Pedagogía Social: Revista Interuniversitaria, núm. 25, España: Sociedad Iberoamericana de Pedagogía Social.

Navarro-Pérez, José Javier et al. (2016), “La justicia juvenil en España: una responsabilidad colectiva Propuestas desde el Trabajo Social”, en Interacción y perspectiva: Revista de Trabajo Social, vol. 6, núm. 2, Venezuela: Universidad del Zulia.

Navarro-Pérez, José Javier y Pastor-Seller, Enrique (2017), "Factores dinámicos en el comportamiento de delincuentes juveniles con perfil de ajuste social. Un estudio de reincidencia”, en Psychosocial Intervention, vol. 26, núm. 1, España: Colegio Oficial de Psicólogos de Madrid. 
Olszewski-Kuwilius, Paula et al. (2014), "Family environment and social development in gifted students”, en Gifted Child Quarterly, vol. 58, núm. 3, Estados Unidos: Sage.

Pérez-Agote, José (2010), "Los retos del proceso de socialización en los sistemas educativos de las sociedades modernas avanzadas", en Politica y sociedad, vol. 47, núm. 2, España: Universidad Complutense de Madrid.

Rodríguez-Costas, Dámaso (1999), “Tribus urbanas violentas”, en Estudios de Ciencia Policial, vol. 51, España: Instituto de Estudios de la Policía.

Ryan, Joseph et al. (2014), "First-Time Violent Juvenile Offenders: Probation, Placement and Recidivism”, en Social Work Research, vol. 38, núm. 1, Estados Unidos: National Association of Social Workers Press. Disponible en: https://www.researchgate.net/ publication/266853373_First-Time_Violent_Juvenile_Offenders_Probation_Placement_and_Recidivism [20 de octubre de 2017].

San Juan, César et al. (2009), "Menores infractores y consumo de drogas: perfil psicosocial y delictivo”, en Criminalidad, vol. 51, núm. 1, Colombia: Policía Nacional de Colombia.

Shifflet-Chila, Erica et al. (2016), "Adolescent and family development: Autonomy and identity in the digital age", en Children \& Youth Services Review, vol. 70, Reino Unido: Elsevier.

Son, Esther et al. (2014), "Peer victimization among young children with disabilities: Early risk and protective factors", en Exceptional Children, vol. 80, núm. 3, Estados Unidos: Council for Excepcional Children.

Suárez-Bautista, Joaquín (2009), "Una sociedad adolescente: Adultos no tan maduros", en Critica, núm. 963, México: Instituto de Investigaciones Filosóficas de la Universidad Nacional Autónoma de México.

Sündermann, Oliver et al. (2014), "Social networks and support in first-episode psychosis: exploring the role of loneliness and anxiety", en Social Psychiatry Psychiatric Epidemiology, vol. 49, Alemania: Dr. Dietrich Steinkopff Verlag.

Tarín, Manuel y Navarro, José (2006), Adolescentes en riesgo. Casos prácticos y estrategias de intervención socioeducativa, España: Editorial CCS.

Vega, Armando et al. (2012), "Adolescentes y jóvenes: desde las conductas de riesgo a la inclusión social”, en Zerbitzuan: Gizarte Zerbitzuetarako Aldizkaria, núm. 52, España: Instituto Vasco de Servicios Sociales.

Villanueva, Lidón et al. (2008), "El acoso escolar y el autoconcepto de agresores, defensores de la víctima y público implicado", en International Journal of Developmental and Educational Psychology, núm. 1, España: Asociación Nacional de Psicología Evolutiva y Educativa de la Infancia, Adolescencia y Mayores.

Wadesango, Newman y Machingambi, Severino (2011), "Causes and Structural Effects of Student Absenteeism: A Case Study of Three South African Universities”, en Journal Social Sciences, vol. 26, núm. 2, Kuwait: Academic Publication Council.

Wimmer, Roger y Dominick, Joseph (1996), La investigación cientifica de los medios de comunicación: una introducción a sus métodos, España: Bosch Editores.

Young, Keneth (2007), "Cognitive behavior therapy with Internet addicts: treatment outcomes and implications”, en Cyberpsychology Behaviour, vol. 10, Estados Unidos: Mary Ann Liebert. 


\section{Anexo}

\section{Tabla 1}

\section{Codificación, sector y entrevistados}

\begin{tabular}{|c|c|c|c|}
\hline Sector & Referencia & Institución de origen & Código \\
\hline \multirow{8}{*}{$\begin{array}{l}\text { Sistema de } \\
\text { Justiciay y } \\
\text { Seguridad }\end{array}$} & Fiscal Justicia Juvenil & Fiscalía provincial de Menores & FJJ \\
\hline & Secretario Judicial & Juzgado de Menores & SJJM \\
\hline & Educador Social & Equipo Técnico Juzgados & $\begin{array}{l}\text { ESETJ1 } \\
\text { EDETJ1 }\end{array}$ \\
\hline & Pedagoga & Equipo Técnico Juzgados & PETJ \\
\hline & Educadora Social & Medidas Judiciales & EMJMA1 \\
\hline & Agente Policía & Grupo Menores Policía & GRUME \\
\hline & Psicóloga & Institución Educativa Reforma & PSIER \\
\hline & Maestro Taller & Institución Educativa Reforma & MTIER \\
\hline \multirow{5}{*}{$\begin{array}{l}\text { Sistema } \\
\text { Educativo }\end{array}$} & Psicopedagogo & Instituto Público Educación & PIPE \\
\hline & Maestro & Instituto Público Educación & MIPE1 \\
\hline & Maestra & Instituto Público Educación & MIPE2 \\
\hline & Orientador & Instituto Público Educación & OIPE \\
\hline & $\begin{array}{l}\text { Monitora Extraesco- } \\
\text { lares }\end{array}$ & Instituto Público Educación & MEIPE \\
\hline \multirow{7}{*}{$\begin{array}{l}\text { Sistema de } \\
\text { Servicios de } \\
\text { Bienestar }\end{array}$} & Educador Social & Servicios Sociales Base & ESSSB \\
\hline & Trabajadora Social & Servicios Sociales Base & TSSSB1 \\
\hline & Psicólogo & Servicios Sociales Base & PSSB2 \\
\hline & Trabajadora Social & $\begin{array}{l}\text { Servicios Sociales Especializa- } \\
\text { dos }\end{array}$ & TSSSE \\
\hline & Trabajador Social & Institución Acogida Infancia & TSIAI \\
\hline & Educadora Social & Institución Acogida Infancia & ESIAI \\
\hline & Educador Prevención & Servicios Sociales Base & EPSSB \\
\hline
\end{tabular}

Fuente: Elaboración propia. 


\section{Tabla 2}

\section{Codificación de relatos a efectos interpretativos}

\begin{tabular}{ccl}
\hline Código & Edad & \multicolumn{1}{c}{ Sexo } \\
\hline 1 & 20 & Mujer \\
\hline 2 & 16 & Hombre \\
\hline 3 & 19 & Mujer \\
\hline 4 & 17 & Hombre \\
\hline 5 & 18 & Mujer \\
\hline 6 & 17 & Hombre \\
\hline 7 & 16 & Mujer \\
\hline 8 & 21 & Hombre \\
\hline
\end{tabular}

Fuente: Elaboración propia.

\section{Figura 1}

\section{Dinámicas de apreciación afectiva (óptica de los ACL)}

$\mathrm{Ns} / \mathrm{Nc}$

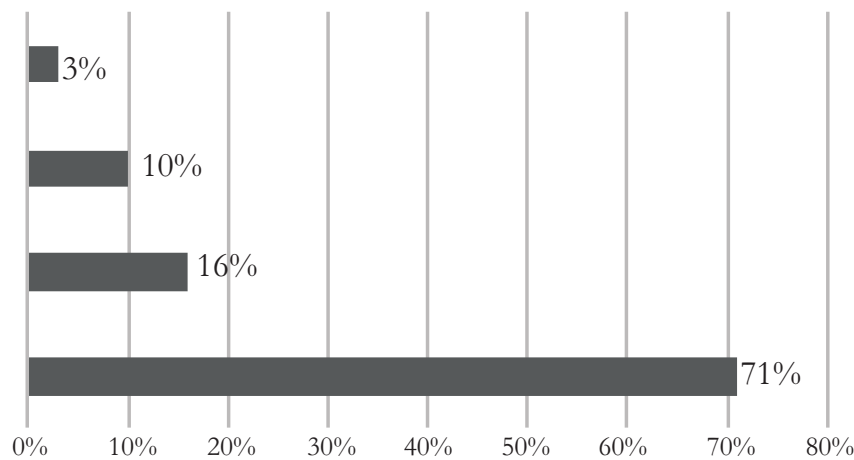

Fuente: Elaboración propia. 


\section{Figura 2}

\section{Patrimonios estimulantes para los ACL con perfil de ajuste}

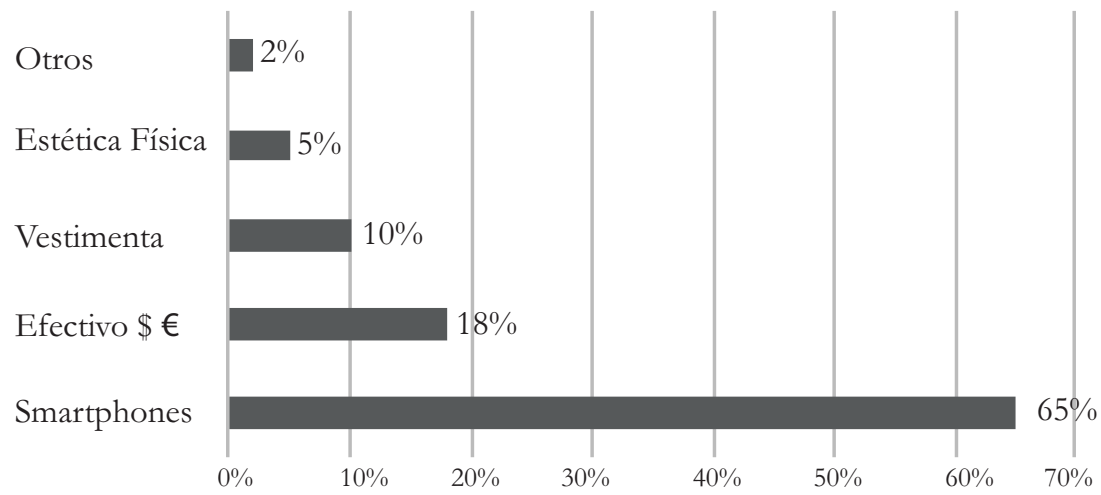

Fuente: Elaboración propia.

\section{Figura 3}

\section{Implicación en actividades de recreo}

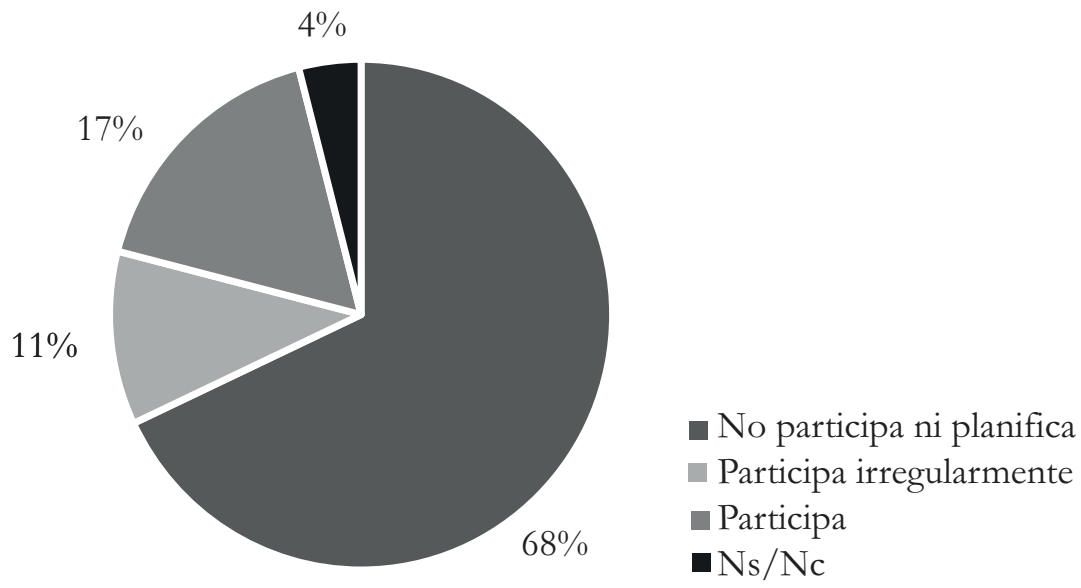

Fuente: Elaboración propia. 
Figura 4

Uso y abuso de viejas/nuevas dependencias por ACL con perfil de ajuste

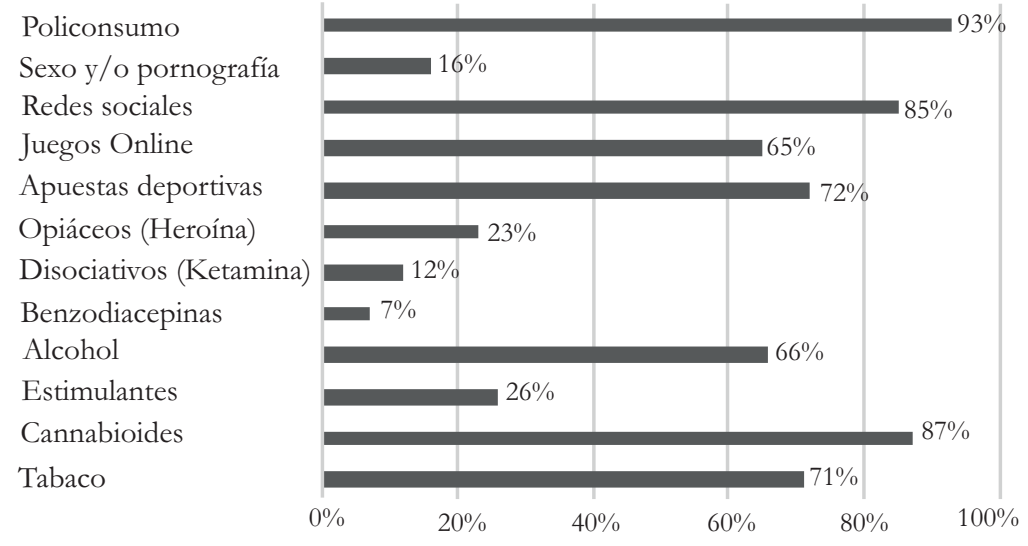

Fuente: Elaboración propia.

José Javier Navarro-Pérez. Doctor en Desarrollo Local y Territorio. Profesor Contratado Doctor del Departamento de Trabajo Social y Servicios Sociales e Investigador del Instituto Interuniversitario de Desarrollo Local de la Universidad de Valencia, España, Grupo GESinn. Líneas de investigación: infancia, adolescencia y juventud, delincuencia juvenil y contextos socioeducativos, participación y territorio. Publicaciones recientes: Navarro, J. J. y Mestre, María Vicenta, El marco global de atención al menor, Valencia: Tirant lo Blanch (2015); Navarro, J. J. y Galiana, Laura, "Prevención del conflicto escolar en primer ciclo de primaria”, en Prisma Social: Revista de Ciencias Sociales, núm. 15 (2015); Uceda, F. X, Navarro, J. J. y Pérez-Cosín, Josep Vicent, "Adolescentes y drogas: su relación con la delincuencia”, en Revista de Estudios Sociales, núm. 58 (2016).

Enrique Pastor-Seller. Doctor en Sociología. Profesor Titular de la Universidad de Murcia, España. Facultad de Trabajo Social. Departamento de Sociología y Trabajo Social. Director del Grupo de Investigación: "Trabajo Social y Servicios Sociales". Líneas de investigación: bienestar social, políticas sociales, participación ciudadana, servicios sociales municipales, educación superior, 
desarrollo local, mediación. Publicaciones recientes: Pastor, E., Támez, G. y Sáenz, K. [eds.], Gobernabilidad, ciudadania y democracia participativa: análisis comparado España-México, Madrid: Dykinson (2014); Pastor, E. y Sánchez, M., "Analysis and Impact of the Economic Crisis and Regulatory Changes in the Needs and Benefits System Municipal Social Services: Analysis Case of Murcia-Spain”, en Revista de Cercetare si Interventie Sociala, núm. 47 (2014); Pastor, E., "Social Work and local community development in the 21st century”, en Arbor: ciencia, pensamiento y cultura, vol. 771 (2015).

Recepción: 22 de mayo de 2017.

Aprobación: 08 de octubre de 2017. 
